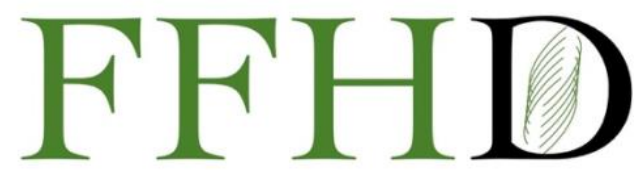

Functional Foods in Health and Disease

\title{
The effect of vitamin D deficiency state on oxidized low- density lipoprotein alteration in patients with type 2 diabetes
}

\author{
Salome Sadat Salehi ${ }^{1}$, Sahar Karimpour ${ }^{1}$, Soghra Rabizadeh ${ }^{1}$, Alireza Esteghamati ${ }^{1}$, Armin \\ Rajab $^{1}$, Sousan Sadat Salehi ${ }^{1}$, Manouchehr Nakhjavani ${ }^{1 *}$, Hossein Mirmiranpour ${ }^{1 * *}$
}

${ }^{1}$ Endocrinology and Metabolism Research Center (EMRC), Vali-Asr Hospital, Imam Khomeini Hospital Complex, Tehran University of Medical Sciences, Tehran, Iran

**First corresponding Author: Hossein Mirmiranpour, MD,PhD, Endocrinology and Metabolism Research Center (EMRC), Vali-Asr Hospital, Tehran University of Medical Sciences, Tehran, Iran

*Second corresponding Author: Manouchehr Nakhjavani, M.D, Endocrinology and Metabolism Research Center (EMRC), Vali-Asr Hospital, Tehran University of Medical Sciences, Tehran, Iran

Submission Date: June $7^{\text {th }}, 2021$; Acceptance Date: July 27 th, 2021 ; Publication Date: August $13^{\text {th }}, 2021$

Please cite this as: Salehi S.S., Karimpour S., Rabizadeh S., Esteghamati A., Rajab A., Salehi S.S., Nakhjavani M., Mirmiranpour $\mathrm{H}$. The effect of vitamin D deficiency state on oxidized low-density lipoprotein alteration in patients with type 2 diabetes. Functional Foods in Health and Disease 2021. 11(8): 357-367. DOI: https://www.doi.org/10.31989/ffhd.v11i8.815

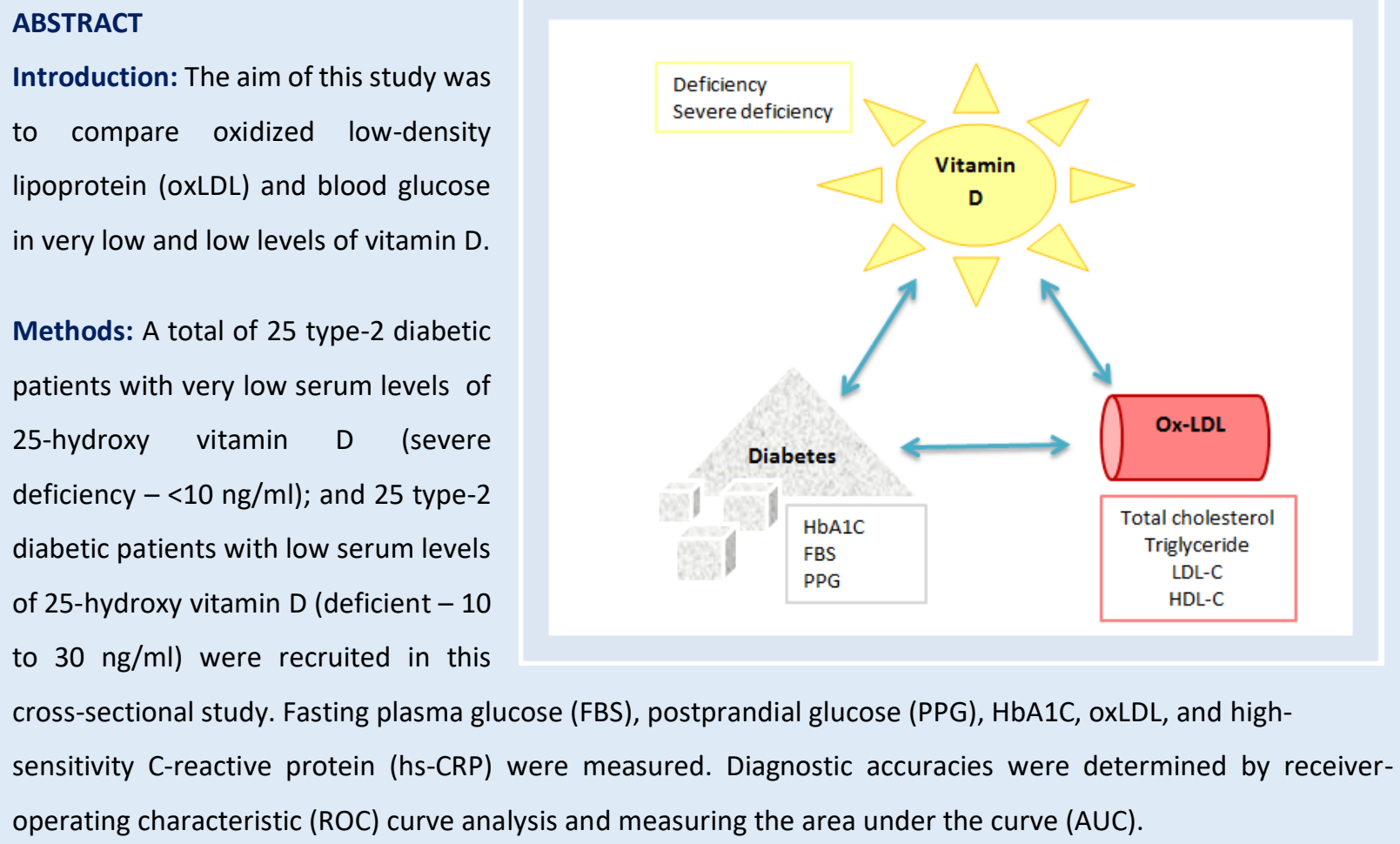

to $30 \mathrm{ng} / \mathrm{ml}$ ) were recruited in this cross-sectional study. Fasting plasma glucose (FBS), postprandial glucose (PPG), HbA1C, oxLDL, and highsensitivity C-reactive protein (hs-CRP) were measured. Diagnostic accuracies were determined by receiveroperating characteristic (ROC) curve analysis and measuring the area under the curve (AUC). 
Results: Patients with severe vitamin D deficiency had higher FBS, PPG, HbA1C, and oxLDL than the vitamin D deficient group $(P<0.05)$. We showed that the AUC of FBS, PPG, HbA1C, and oxLDL were in the range of 0.7-0.9, which indicates moderate accuracies in differentiating severe vitamin D deficiency from deficiency condition.

Conclusion: In conclusion, diabetic patients with severe vitamin D deficiency had higher FBS, PPG, and oxLDL levels compared with deficient ones. ROC curve analysis of FBS, PPG, HbA1C, and oxLDL showed these markers are independent predictors of severe vitamin D deficiency and significantly discriminate between very low and low levels of vitamin D deficiency in patients with diabetes.

Keywords: Type 2 diabetes mellitus. Vitamin D deficiency. OxLDL. Fasting blood glucose.

(CFFC 2021. This is an Open Access article distributed under the terms of the Creative Commons Attribution 4.0 License (http://creativecommons.org/licenses/by/4.0)

\section{INTRODUCTION}

Hypovitaminosis D has been recently recognized as a worldwide epidemic. In type 2 diabetes, the prevalence of vitamin D deficiency is $20 \%$ higher than in non-diabetics [1]. Low vitamin D status can be caused by several factors, including insufficient cutaneous synthesis (due to limited sunlight exposure or aging), inadequate intake and absorption of vitamin D, or obesity [2]. Vitamin D can be used as a profile marker for cardiovascular disease (CVD) in view of the fact that vitamin $D$ deficiency plays an essential role in the genesis of coronary risk factors and CVD and seems to be linked to predisposal to hypertension, diabetes, and metabolic syndrome [35]. Vitamin $D$ is thought to have both direct (by the activation of the vitamin D receptors) and indirect (by the regulation of calcium homeostasis) effects on various mechanisms related to the pathophysiology of type 2 diabetes [6-8]. The effect of vitamin D on pancreatic cells and subsequent insulin release is mediated through vitamin D receptor [8-10]. Vitamin D deficiency impairs insulin secretion of pancreatic cells and increases insulin resistance, which are major factors in the pathogenesis of type 2 diabetes [10].

Oxidized low-density lipoprotein (oxLDL), derived from LDL-cholesterol under oxidative stress such as hyperglycemia, plays a key role in diabetes complications and atherosclerosis [11]. OxLDL and its components have many proinflammatory and proatherogenic properties [12]. Macrophage uptake of oxLDL is thought to play a central role in foam cell formation and pathogenesis of atherosclerosis [13]. Active vitamin $D$ suppresses foam cell formation by reducing oxLDL cholesterol uptake in comparison with vitamin D-deficient macrophages [14]. Creactive protein (CRP) is an acute-phase protein that participates in the innate immune response and it has been shown to be a reliable measure of underlying systemic inflammation $[12,15]$. Active vitamin D diminishes the expression of proinflammatory cytokines which can induce production of CRP [16].

Here we aimed to study the effects of different levels of vitamin D hypovitaminosis on blood glucose, oxLDL, and hs-CRP in two groups of diabetic patients, 
defined as patients with severe vitamin D deficiency and patients with vitamin D deficiency.

\section{MATERIAL AND METHODS}

This is a descriptive cross-sectional study on 50 patients with type 2 diabetes referring to the diabetes clinic of Vali-asr Hospital, affiliated with Tehran University of Medical Sciences. A total of 25 type-2 diabetic patients with very low serum levels of 25hydroxy vitamin D (severe deficiency $-<10 \mathrm{ng} / \mathrm{ml}$ ) and 25 type- 2 diabetic patients with low serum levels of 25-hydroxy vitamin D (deficiency - 10-30 ng/ml) were recruited [4]. The sample size was estimated based on the proper sample size formula [17] in according to a pilot study, and a value of $\alpha=0.05$ and $\beta=0.2$ were used as input parameters for calculation. Two groups were matched according to age, sex, and body mass index (BMI). The exclusion criteria in the study were the following: suffering from underlying medical conditions including malignancy, acute infection, advanced heart failure, renal and liver failure, and also pregnancy. Diabetes mellitus was diagnosed according to the criteria of the American Diabetes Association [18]. Demographic and anthropometric data, including age, sex, duration of diabetes, height, weight in light clothing, and blood pressure (in sitting position after 10 min rest) were recorded. Blood pressure was re-measured twice after $5 \mathrm{~min}$ and averaged. The body mass index (BMI; $\mathrm{kg} / \mathrm{m} 2$ ), was calculated according to the Quetelet formula. Antidiabetic and lipid-lowering drug history was taken and recorded.

Measurements: The following blood sample measurements were conducted regarding the routine processes of our clinic [19]. Venous blood was taken at 08:00 am after 12 hours of fasting. The blood was obtained by direct puncture to the antecubital vein while the subjects were in supine rest for 30 minutes. Venous blood was collected into ethylene diamine tetraacetic acid-containing $(1.5 \mathrm{mg} / \mathrm{ml})$ tubes and placed on ice immediately. The obtained plasma was centrifuged at $3000 \mathrm{rpm}$ for $15 \mathrm{~min}$ at $4^{\circ} \mathrm{C}$ and then kept at $-70 \circ \mathrm{C}$ until analysis. Serum levels of total cholesterol, HDL-C, LDL-C, and triglycerides (TG) were measured by direct colorimetric enzymatic methods (Parsazmun. Karaj, Iran). OxLDL was determined using a sandwich enzyme-linked immunosorbent assay (ELISA) (Mercodia, Uppsala, Sweden). Intra and interassay coefficients of variation (CV) were 4.5 and 7\%, respectively, and the detection limit was $\leq 0.03 \mathrm{U} / \mathrm{L}$. Glucose measurements (intra-assay CV 2.1\%, interassay CV 2.6\%) were carried out using the glucose oxidase method. HbA1C was estimated by HighPerformance Liquid Chromatography (HPLC) Method. Insulin was measured (intra-assay CV $4.7 \%$, interassay CV 3.3\%) by the immune radiometric method (Immunotech, Finland). Hs-CRP was assessed using a two-site, enzyme-linked immunosorbent assay (ELISA) (Diagnostic Biochem, London, Ontario, Canada). The sensitivity of the assay was $10 \mathrm{ng} / \mathrm{L}$. Intra- and inter-assay coefficients of variation (CV) were $8 \%$ and $10 \%$, respectively. Creatinine was measured using the calibrated Jaffe method (Parsazmoon, Karaj, Iran, intra-assay CV = 3.3\%). Blood urea was measured by enzymatic colorimetric method. Glomerular filtration rate (GFR) was calculated with the Cockroft-Gault formula. Serum 25(OH) Vitamin D was determined using the enzymelinked immunosorbent assay technique (DIAsource, Louvain-la-Neuve, Belgium). Intra- and inter-assay coefficients of variation (CV) were 4.7 and $5.7 \%$, respectively. 
Statistical Analysis: Data was analyzed using SPSS software (version16.0; spss, Chicago, Illinois, USA). Shapiro-Wilk test was employed to test the normality of the variables in each group. The continuous variables are expressed as means \pm standard deviation (SD). Quantitative variables were compared between the two groups using $t$-test and Mann-Whitney test. The qualitative variables were compared with chisquare test. Pearson's correlation test was employed to study correlations among variables. Receiver operating characteristics (ROC) curve was recruited for sensitivity/specificity analysis. For the area under the curve $(A \cup C)$ of the ROC curve, $0.5<A \cup C \leq 0.7$ was less accurate, $0.7<A U C \leq 0.9$ was moderately accurate, $0.9<A U C<1.0$ was very accurate, and $A U C=1$ was perfectly accurate [20]. $\mathrm{P}$ values $<0.05$ were considered statistically significant.

Ethics: The research was carried out according to the principles of the Declaration of Helsinki. The local ethics review committee of Tehran University of Medical Sciences approved the study protocol. All participants gave written informed consent before participation.

\section{RESULTS}

The demographic and biochemical characteristics of the participants are illustrated in Table 1. There was no significant difference between groups regarding age, gender, BMI, waist circumference, duration of diabetes, systolic and diastolic blood pressure, hsCRP, creatinine, GFR, total cholesterol, LDL-C, HDL-C, triglycerides, and medications (antidiabetic and lipid- lowering agents). The mean value of vitamin D level was $7.67 \pm 3.10 \mathrm{ng} / \mathrm{dl}$ in group with severe vitamin D deficiency and $21.20 \pm 6.67 \mathrm{ng} / \mathrm{dl}$ in deficient group. FBS, PPG and $\mathrm{HbA} 1 \mathrm{C}$ were significantly higher in severe vitamin $D$ deficiency $(p<0.05)$. Also the group with severe vitamin D deficiency had significantly higher oxLDL levels compared to the deficient group (58.07 $\pm 16.29 \mathrm{U} / \mathrm{dL}$ vs. $48.43 \pm 12.66 \mathrm{U} / \mathrm{dL}, \mathrm{p}<0.05)$ (Figure 1).

ROC analysis was performed in the study to assess the predictive value of FBS, PPG, $\mathrm{HbA} 1 \mathrm{C}$ and oxLDL for diagnosis of vitamin $D$ severe deficiency condition (Table 2 and Figure 2). The area under curve (AUC) was 0.86 for FBS, 0.83 for PPG, 0.75 for $\mathrm{HbA} 1 \mathrm{C}$, and 0.71 for oxLDL $(p<0.05)$. Particular attention should be paid to FBS for which AUC in the presence of severe vitamin $D$ deficiency was 0.86 with a cut-off value of $>154.5 \mathrm{mg} / \mathrm{dl}, 67.00 \%$ sensitivity and $65.00 \%$ specificity. We also showed a very high diagnostic value of PPG determination (AUC $0.83, p<0.05$ ) in differentiating the group of diabetic patients with severe vitamin D deficiency from the deficient group. The cut-off value was $>219.5 \mathrm{mg} / \mathrm{dl}$ with a sensitivity of $72.00 \%$ and specificity of $75.00 \%$. When $\mathrm{HbA} 1 \mathrm{C}$ $>7.9 \%$, the sensitivity and the specificity are $72.00 \%$ and $75.00 \%$ and when oxLDL $>50.5 \mathrm{U} / \mathrm{dl}$, the sensitivity and the specificity are $67.00 \%$ and $70.00 \%$, respectively, in the differentiation of severe vitamin $D$ deficiency from deficiency condition.

There was no significant correlation between vitamin D levels with each variable. Similarly, there was not any association among variables in both vitamin D deficiency and severe deficiency groups. 
Table 1. Baseline Characteristics of study population.

\begin{tabular}{|c|c|c|c|}
\hline & $\begin{array}{l}\text { Vitamin D deficient } \\
(<10 \mathrm{ng} / \mathrm{ml})\end{array}$ & $\begin{array}{l}\text { Vitamin D insufficient } \\
(10-30 \mathrm{ng} / \mathrm{ml})\end{array}$ & P-value \\
\hline Gender (male, female) & $(13,12)$ & $(7,18)$ & NS \\
\hline Age (years) & $56.56 \pm 11.79$ & $55.60 \pm 14.20$ & NS \\
\hline BMI $\left(\mathrm{kg} / \mathrm{m}^{2}\right)$ & $28.32 \pm 4.47$ & $27.54 \pm 3.37$ & NS \\
\hline Waist circumference $(\mathrm{cm})$ & $94.08 \pm 10.76$ & $93.84 \pm 9.33$ & NS \\
\hline Duration of diabetes (year) & $9.42 \pm 6.4$ & $9.65 \pm 5.31$ & NS \\
\hline \multicolumn{4}{|l|}{ Blood pressure (mmHg) } \\
\hline SBP & $127.76 \pm 21.94$ & $132.16 \pm 20.14$ & NS \\
\hline DBP & $79.92 \pm 11.63$ & $76.12 \pm 12.80$ & NS \\
\hline MAP & $95.86 \pm 14.20$ & $94.80 \pm 9.33$ & NS \\
\hline \multicolumn{4}{|l|}{ Plasma glucose (mg/dl) } \\
\hline $\mathrm{FBS}(\mathrm{mg} / \mathrm{dl})$ & $199.61 \pm 64.74$ & $146.78 \pm 46.40$ & $<0.01$ \\
\hline PPG (mg/dl) & $284.1 \pm 81.4$ & $199.83 \pm 40.74$ & $<0.01$ \\
\hline $\mathrm{HbA} 1 \mathrm{C}(\%)$ & $8.37 \pm 1.81$ & $6.93 \pm 1.33$ & $<0.01$ \\
\hline Insulin $(\mu \mathrm{u} / \mathrm{ml})$ & $22.38 \pm 15.21$ & $18.19 \pm 12.06$ & NS \\
\hline Hs-CRP ( mg/ml) & $4.23 \pm 0.43$ & $3.09 \pm 0.24$ & NS \\
\hline Creatinine (mg/dl) & $.96 \pm 0.22$ & $.99 \pm 0.22$ & NS \\
\hline GFR (mL/min) & $84.11 \pm 27$ & $86.45 \pm 27.95$ & NS \\
\hline Urea $(\mathrm{g} / 24 \mathrm{~h})$ & $27.13 \pm 13.53$ & $30.3 \pm 16.72$ & NS \\
\hline Uric Acid (mg/dl) & $5.27 \pm 1.07$ & $5.23 \pm 1.25$ & NS \\
\hline \multicolumn{4}{|l|}{ Plasma cholesterol (mg/dl) } \\
\hline Total cholestrol & $187.38 \pm 29.72$ & $190.78 \pm 52.72$ & NS \\
\hline LDL-C (mg/dl) & $106.05 \pm 27.96$ & $108.91 \pm 42.95$ & NS \\
\hline $\mathrm{HDL}-\mathrm{C}(\mathrm{mg} / \mathrm{dl})$ & $43.75 \pm 9.71$ & $44.13 \pm 10.86$ & NS \\
\hline Triglyceride (mg/dl) & $194.67 \pm 86.82$ & $155.95 \pm 70.51$ & NS \\
\hline OxLDL (U/dl) & $58.07 \pm 16.29$ & $48.43 \pm 12.66$ & 0.04 \\
\hline Vitamin D (ng/dl) & $7.67 \pm 2.10$ & $21.20 \pm 6.67$ & $<0.001$ \\
\hline \multicolumn{4}{|l|}{ Medication - no. (\%) } \\
\hline Insulin only & $1(50)$ & $1(50)$ & NS \\
\hline OAD only & $21(50)$ & $21(50)$ & NS \\
\hline OAD + insulin & $1(33.3)$ & $2(66.7)$ & NS \\
\hline Statin & $12(54.5)$ & $10(45.5)$ & NS \\
\hline
\end{tabular}

Data is presented as mean \pm SD or number and percent. $P$ value $<0.05$ is considered statistically significant.

Abbreviations: BMI: body mass index, SBP: systolic blood pressure, DBP: diastolic blood pressure, FBS: fasting blood sugar, PPG: postprandial glucose, HbA1C: hemoglobin A1C, Hs-CRP: High sensitivity C-reactive protein, GFR: Glomerular filtration rate, LDL-C: low density lipoprotein cholesterol, HDL-C: high density lipoprotein cholesterol, TG: Triglyceride, oxLDL: oxidase LDL, OAD: Oral antidiabetic drug, NS: non-significant. 
Table 2. Receiver Operating Characteristic (ROC) analysis for blood glucose, lipids and hs-CRP in differentiating between vitamin D deficiency and severe deficiency

\begin{tabular}{|c|c|c|c|c|c|c|}
\hline & AUC & $\begin{array}{l}95 \% \text { confidence } \\
\text { interval }\end{array}$ & p-value & Sensitivity \% & Specificity \% & Cut-off value \\
\hline Hs-CRP & 0.66 & $(0.49-0.83)$ & 0.11 & ----- & ----- & ----- \\
\hline TG & 0.67 & $(0.49-0.86)$ & 0.10 & ----- & ----- & ----- \\
\hline HDL-C & 0.46 & $(0.26-0.66)$ & 0.71 & ----- & ----- & ----- \\
\hline LDL-C & 0.46 & $(0.27-0.64)$ & 0.68 & ----- & ----- & ----- \\
\hline OxLDL & 0.71 & $(0.52-0.88)$ & 0.04 & 67 & 70 & 50.5 \\
\hline FBS & 0.86 & $(0.74-0.98)$ & $<0.001$ & 67 & 65 & 154.5 \\
\hline PPG & 0.83 & $(0.69-0.98)$ & $<0.001$ & 72 & 75 & 219.5 \\
\hline HbA1C & 0.75 & $(0.57-0.92)$ & $<0.01$ & 72 & 75 & 7.9 \\
\hline
\end{tabular}

AUC: Area under the curve (Cl 95\%)

Abbreviations: Hs-CRP: High sensitivity C-reactive protein, TG: Triglyceride, HDL-C: high density lipoprotein cholestrol LDL-C: low density lipoprotein cholesterol, ox LDL: oxidase LDL, FBS: fasting blood sugar, PPG: postprandial glucose, HbA1C: hemoglobin A1C.

\section{DISCUSSION}

Our findings demonstrated that diabetic patients with severe vitamin D deficiency had significantly higher oxLDL levels compared to the deficient group (Figure 1). OxLDL is a particle derived from circulating $L D L$ under oxidative stress in the subendothelial space of the arterial wall, and a small amount of oxLDL may also be released into circulation [11, 21-23]. The source of such oxLDL could be the direct release of modified LDL from ruptured or permeable plaques or ischemic injury due to damaged cell membranes. It is likely that elevated levels of oxLDL reflect the turnover of oxLDL in newly formed or progressing lesions in arteries [24]. Vitamin D can reduce the damage of superoxide-mediated stress in a dose- and time-dependent manner [25]. Riek et al. study has provided evidence that vitamin $D$ receptors activation by vitamin $D$ decreases macrophage oxLDL uptake by reducing gene expression in patients with diabetes [14]. Similarly, Jisu et al. showed that diabetic-derived macrophages cultured in $1,25(\mathrm{OH}) 2$ D3supplemented media had decreased oxLDL cholesterol uptake both qualitatively and quantitatively by 40 and $50 \%$, respectively, when compared to macrophages cultured in vitamin Ddeficient media [1]. It is worth mentioning that, despite the significant difference in oxLDL, we found no difference in total cholesterol, HDL-C, LDL-C, and triglycerides between vitamin D deficiency levels. Furthermore, we showed that there were no direct associations between oxLDL with other lipid markers. The oxidation of LDL is a complex process during which not only the lipids but also the proteins undergo oxidative changes [26]. In agreement, some previous studies demonstrated that a change in the oxLDL level certainly does not occur in parallel with a change in the LDL-cholesterol level in hyperglycemic conditions $[11,27]$. In vitamin D deficiency, the oxidative-antioxidative balance was observed to change in favor of oxidative stress [28]. Uberti et al. study showed that administering Vitamin $D$ to endothelial cells before induction of oxidative stress can improve cell viability [29]. During hyperglycemia or an insulin-resistant state, increased scavenger receptor expression promotes the foam-cell formation and is considered a link between diabetes and atherosclerosis [13]. 


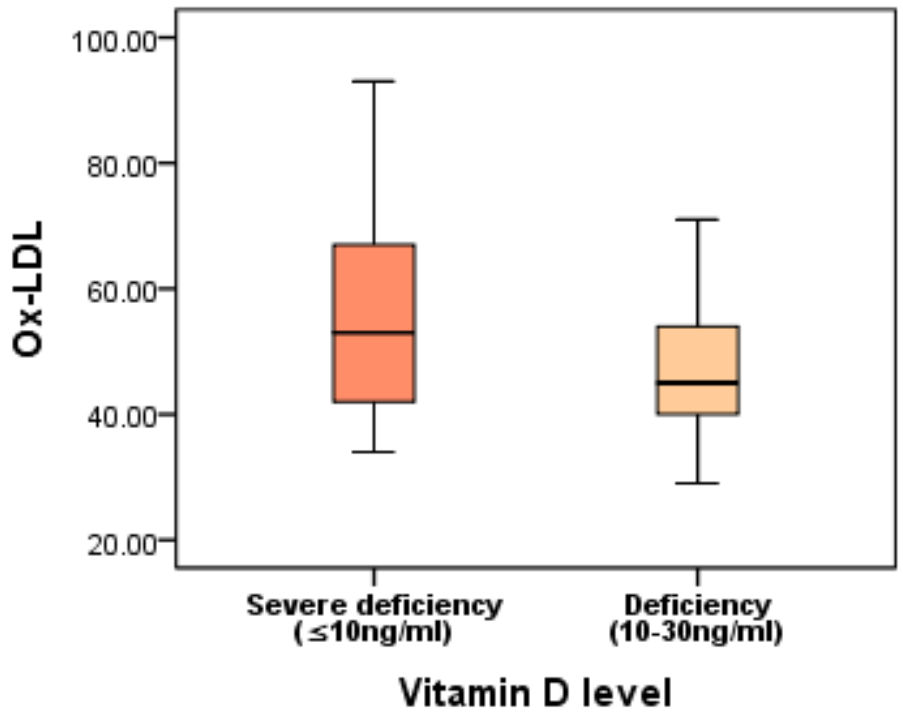

Figure 1. Boxplots showing distributions of oxidized LDL in different levels of vitamin D deficiency in patients with type 2 diabetes.

Although our study groups were the same with respect to anthropometric data, duration of diabetes and lipid and glucose lowering medications, we found significantly higher serum FBS, PPG and $\mathrm{HbA} 1 \mathrm{C}$ in severe vitamin $D$ deficiency in type 2 diabetes. Our results are generally consistent with previous reports $[2,30]$. However, further interventional studies would be needed to show the biological effects of vitamin $D$ deficiency on glycemic control in type 2 diabetes mellitus; it has been shown that 25(OH)D deficiency may decrease insulin secretion and increase peripheral insulin resistance and blood sugar (Figure 2) [31]. With regard to glucose homeostasis, vitamin D affects pancreatic beta-cell proliferation and survival and its ability to respond to situations of increased insulin demand, such as in type 2 diabetes [2]. In vivo experiments showed impaired insulin secretion in vitamin D-deficient rats and clearly established a molecular role of the vitamin D receptor in the endocrine function of the pancreas [32].

In this study there was no significant difference in hs-CRP between the two groups. Similarly, Carlson et al. reported that correction of hypovitaminosis $D$ had no statistically significant effect on hs-CRP levels in patients with hypertension [33]. Our findings are also in accord with Pittas et al. study which there were no statistically significant differences in plasma C-reactive protein or interleukin- 6 between the two treatment groups of placebo and vitamin D in non-diabetic adults [34]. Here we found no significant correlation between oxLDL and hs-CRP of two groups. Conversely, Zhang et al. study reported a positive correlation between oxLDL and CRP in patients with diabetes and acute coronary syndrome [15]. CRP is bound to oxidized LDL but not to native LDL and this binding promotes oxLDL uptake by macrophages $[12,35]$. The interaction between oxLDL and CRP is calcium-dependent and, most importantly, occurred at physiological concentrations, i.e., for CRP at 0.1 to $2 \mathrm{mg} / \mathrm{L}$ [35-37]. Macrophages absorb these lipoproteins and transform into foam cells. Thus, this process contributes to higher levels of inflammatory cytokines produced by macrophages and subsequently increases CRP production by the liver; so oxLDL increases CRP mRNA and protein expression [38]. 
We compared the diagnostic accuracies of FBS, PPG, HbA1C, and oxLDL using ROC curve analysis and area under the curve, which is the reflection of how good these markers are at distinguishing between low and very low levels of vitamin $D$ in patients with diabetes.
The AUC of FBS, PPG, HbA1C, and oxLDL were in the range of 0.7-0.9 which indicates their moderate accuracies in differentiating vitamin D deficiency from severe deficiency condition (Figure 3, Table 2).

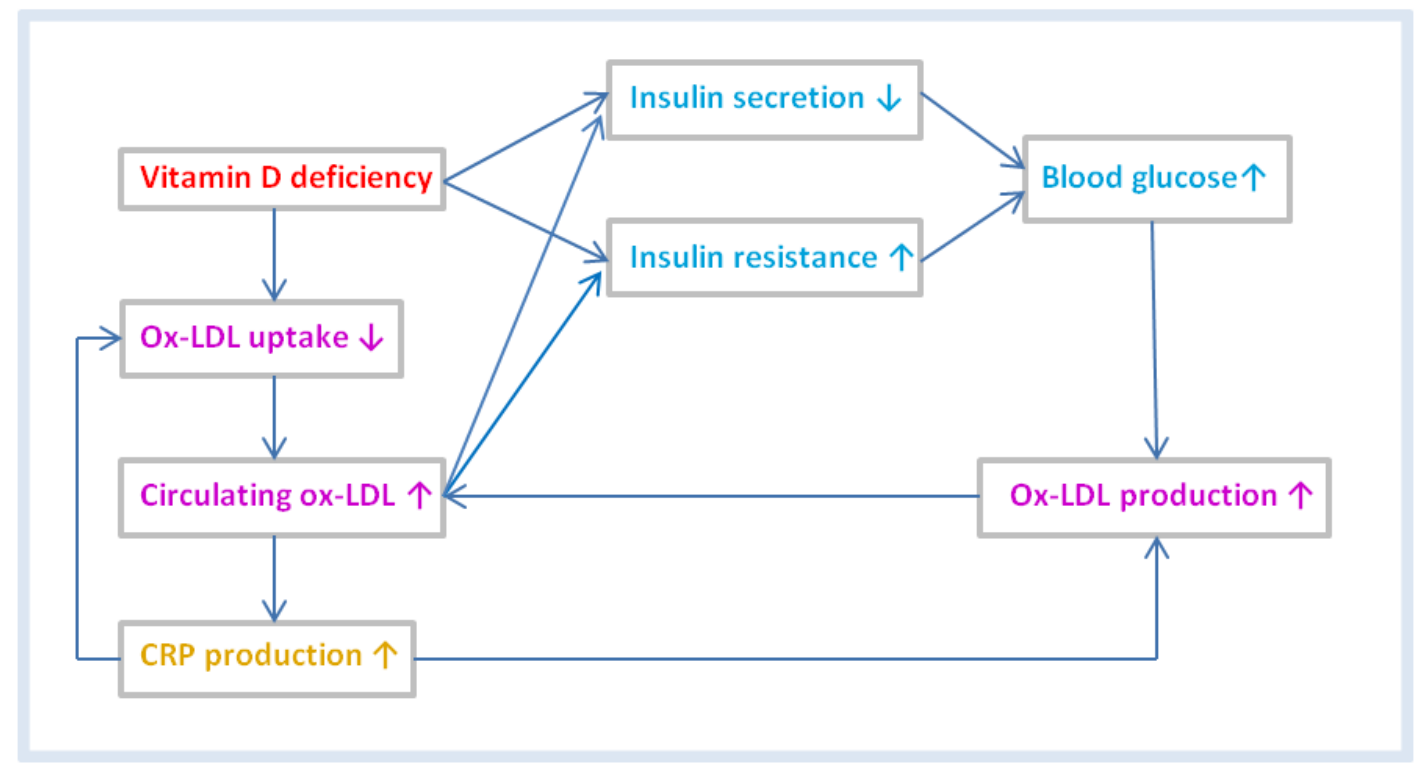

Figure 2. The theoretical framework for the interactions of blood glucose oxidized low-density lipoprotein (ox-LDL) and C-reactive protein (CRP) in vitamin D deficiency. Vitamin D deficiency decreases insulin secretion and increases peripheral insulin resistance and blood glucose. Hyperglycemia as an oxidative stress induces ox-LDL production. On the other hand, vitamin D deficiency directly increases ox-LDL endothelial uptake that results in higher levels of circulating ox-LDL and subsequent CRP production. Increased circulating oxLDL also may decrease insulin secretion and increase peripheral insulin resistance and blood glucose.

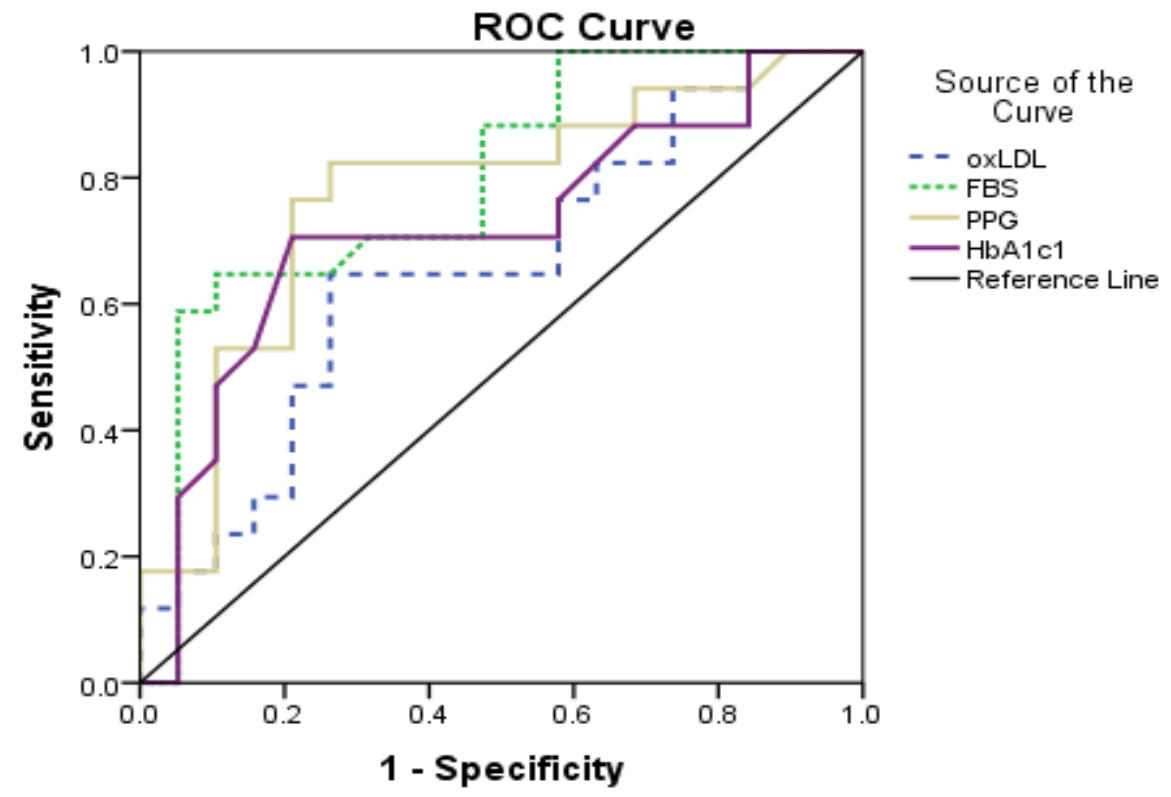

Diagonal segments are produced by ties.

Figure 2. ROC curve of oxLDL, FBS, PPG and HbA1C in vitamin D deficiency. 
To our knowledge, no study compared lipid profiles, blood glucose and markers of inflammation and their predictive values in different levels of vitamin D deficiency.

The principal limitation of this study is its crosssectional nature which precludes the determination of the direction of causality. Findings from studies in Iran have demonstrated that vitamin D deficiency is a highly prevalent problem among Iranians [39, 40]. Further prospective and interventional studies are needed to clarify the effects of vitamin D supplementations on glycemic control and lipid oxidation in patients with type 2 diabetes mellitus.

\section{CONCLUSION}

In conclusion, diabetic patients with severe vitamin D deficiency had higher FBS, PPG, HbA1C, and oxLDL levels compared with deficient ones. The findings presented herein have therapeutic implications. This study hypothesizes that, in patients with diabetes mellitus type 2 , normal levels of vitamin $D$ in the blood may facilitate glucose control. The results also could possibly point to the effect of vitamin $D$ deficiency level on oxLDL production.

List of abbreviations: Hs-CRP: High sensitivity Creactive protein, OxLDL: Oxidized low-density lipoprotein, LDL: Low-density lipoprotein, HDL: Highdensity lipoprotein, BMI: Body mass index, FBS: Fasting blood sugar, PPG: Post prandial glucose, AUC: Area under curve, ROC: Receiver operating characteristics, SBP: Systolic blood pressure, DBP: Diastolic blood pressure.

Funding: This study was funded by grants provided by the Tehran University of Medical Sciences.

Author's contributions: All the authors approved the final version and have the agreement to be responsible for all aspects of the work in confirming that questions related to the accuracy or reliability of any part of the work are appropriately inspected and resolved. HM contributed to the original idea of the paper, doing the experimental work and data collection; AR accompany in assessment of biochemical parameters; MN designed the research; SK treated patients and collected material and clinical data from patients; AE performed the assays; SR analyzed the data; SSS wrote the paper; SoSS performed visualization.

Conflict interest: The authors declare that there is no conflict of interests.

Ethics approval and consent to participate: The study was approved by the Local Ethics Committee of Tehran University of Medical Sciences, based on the Helsinki.

Acknowledgement: The authors of this paper sincerely acknowledge the staff at the endocrine laboratory of Endocrine and Metabolism Research Center (EMRC).

\section{REFERENCES}

1. Oh J, Weng S, Felton SK, Bhandare S, Riek A, Butler B, Proctor BM, Petty M, Chen Z, Schechtman KB et al: $1,25(\mathrm{OH}) 2$ vitamin $d$ inhibits foam cell formation and suppresses macrophage cholesterol uptake in patients with type 2 diabetes mellitus. Circulation 2009, 120(8):687-698.

2. Liu E, Meigs JB, Pittas AG, McKeown NM, Economos CD, Booth SL, Jacques PF: Plasma 25-hydroxyvitamin $d$ is associated with markers of the insulin resistant phenotype in nondiabetic adults. J Nutr 2009, 139(2):329334.

3. Wolf M, Shah A, Gutierrez O, Ankers E, Monroy M, Tamez H, Steele D, Chang Y, Camargo CA, Jr., Tonelli M et al: Vitamin D levels and early mortality among incident 
hemodialysis patients. Kidney international 2007, 72(8):1004-1013.

4. Anderson JL, May HT, Horne BD, Bair TL, Hall NL, Carlquist JF, Lappe DL, Muhlestein JB, Intermountain Heart Collaborative Study G: Relation of vitamin D deficiency to cardiovascular risk factors, disease status, and incident events in a general healthcare population. The American journal of cardiology 2010, 106(7):963-968.

5. Oberoi D, Mehrotra V, Rawat A: "Vitamin D" as a profile marker for cardiovascular diseases. Ann Card Anaesth 2019, 22(1):47-50.

6. Mitri J, Muraru MD, Pittas AG: Vitamin D and type 2 diabetes: a systematic review. European journal of clinical nutrition 2011, 65(9):1005-1015.

7. Kayaniyil S, Vieth $R$, Retnakaran R, Knight JA, Qi Y, Gerstein HC, Perkins BA, Harris SB, Zinman B, Hanley AJ: Association of vitamin D with insulin resistance and betacell dysfunction in subjects at risk for type 2 diabetes. Diabetes care 2010, 33(6):1379-1381.

8. Berridge MJ: Vitamin D deficiency and diabetes. Biochemical Journal 2017, 474(8):1321-1332.

9. Knekt P, Laaksonen M, Mattila C, Harkanen T, Marniemi J, Heliovaara M, Rissanen H, Montonen J, Reunanen A: Serum vitamin D and subsequent occurrence of type 2 diabetes. Epidemiology 2008, 19(5):666-671.

10. Esteghamati A, Fotouhi A, Faghihi-Kashani S, HafeziNejad N, Heidari B, Sheikhbahaei S, Zandieh A, Nakhjavani $M$ : Non-linear contribution of serum vitamin $D$ to symptomatic diabetic neuropathy: A case-control study. Diabetes research and clinical practice 2016, 111:44-50.

11. Nakhjavani M, Khalilzadeh $O$, Khajeali L, Esteghamati A, Morteza A, Jamali A, Dadkhahipour S: Serum oxidized-LDL is associated with diabetes duration independent of maintaining optimized levels of LDL-cholesterol. Lipids 2010, 45(4):321-327.

12. Chang $\mathrm{M}-\mathrm{K}$, Binder $\mathrm{CJ}$, Torzewski M, Witztum JL: Creactive protein binds to both oxidized LDL and apoptotic cells through recognition of a common ligand: phosphorylcholine of oxidized phospholipids. Proceedings of the National Academy of Sciences 2002, 99(20):13043-13048.

13. Laszlo Nagy kPT, k, Jacqueline G. A. Alvarez HC, Evans aRM: oxidized LDL regulates macrophage gene expression through ligand activation of PPARy. cell 1998, 93:229-240.
14. Riek $A E$, Oh J, Bernal-Mizrachi C: Vitamin D regulates macrophage cholesterol metabolism in diabetes. The Journal of steroid biochemistry and molecular biology 2010, 121(1-2):430-433.

15. Zhang Y-c, Wei J-j, Wang F, Chen M-t, Zhang M-z: Elevated levels of oxidized low-density lipoprotein correlate positively with C-reactive protein in patients with acute coronary syndrome. Cell biochemistry and biophysics $2012,62(2): 365-372$.

16. Giulietti A, van Etten E, Overbergh L, Stoffels K, Bouillon R, Mathieu C: Monocytes from type 2 diabetic patients have a pro-inflammatory profile: 1, 25-Dihydroxyvitamin D3 works as anti-inflammatory. Diabetes research and clinical practice 2007, 77(1):47-57.

17. Statulator: An online statistical calculator. Sample Size Calculator for Comparing Two Independent Means. [http://statulator.com/SampleSize/ss2M.html],

18. Association AD: 2. Classification and diagnosis of diabetes: standards of medical care in diabetes -2018 . Diabetes care 2018, 41(Supplement 1):S13-S27.

19. Motamed M, Nargesi AA, Heidari B, Mirmiranpour $H$, Esteghamati A, Nakhjavani M: Oxidized Low-Density Lipoprotein (ox-LDL) to LDL Ratio (ox-LDL/LDL) and ox-LDL to High-Density Lipoprotein Ratio (ox-LDL/HDL). Clin Lab 2016, 62(9):1609-1617.

20. Greiner M, Pfeiffer D, Smith RD: Principles and practical application of the receiver-operating characteristic analysis for diagnostic tests. Preventive veterinary medicine 2000, 45(1-2):23-41.

21. Li D, Mehta JL: Oxidized LDL, a critical factor in atherogenesis. Cardiovascular research 2005, 68(3):353354.

22. Liu M-L, Ylitalo K, Salonen R, Salonen JT, Taskinen M-R: Circulating oxidized low-density lipoprotein and its association with carotid intima-media thickness in asymptomatic members of familial combined hyperlipidemia families. Arteriosclerosis, thrombosis, and vascular biology 2004, 24(8):1492-1497.

23. Xu H, Jiang J, Chen W, Li W, Chen Z: Vascular macrophages in atherosclerosis. Journal of immunology research 2019, 2019.

24. Tsimikas S, Witztum JL: Measuring circulating oxidized low-density lipoprotein to evaluate coronary risk. Circulation 2001, 103(15):1930-1932.

25. Polidoro L, Properzi G, Marampon F, Gravina G, Festuccia C, Di Cesare E, Scarsella L, Ciccarelli C, Zani B, Ferri C: 
Vitamin D protects human endothelial cells from $\mathrm{H} 2 \mathrm{O} 2$ oxidant injury through the Mek/Erk-Sirt1 axis activation. Journal of cardiovascular translational research 2013, 6(2):221-231.

26. Parthasarathy S, Raghavamenon A, Garelnabi MO, Santanam N: Oxidized low-density lipoprotein. Free Radicals and Antioxidant Protocols 2010:403-417.

27. Holvoet P: Relations between metabolic syndrome, oxidative stress and inflammation and cardiovascular disease. Verh K Acad Geneeskd Belg 2008, 70(3):193-219.

28. Baser H, Can U, Baser S, Hidayetoglu BT, Aslan U, Buyuktorun I, Yerlikaya FH: Serum total oxidant/antioxidant status, ischemia-modified albumin and oxidizedlow density lipoprotein levels in patients with vitamin $D$ deficiency. Archives of endocrinology and metabolism 2015, 59(4):318-324.

29. Uberti F, Lattuada D, Morsanuto V, Nava U, Bolis G, Vacca G, Squarzanti D, Cisari C, Molinari C: Vitamin D protects human endothelial cells from oxidative stress through the autophagic and survival pathways. The Journal of Clinical Endocrinology \& Metabolism 2013, 99(4):1367-1374.

30. Gradinaru D, Borsa C, Ionescu C, Margina D, Prada Gl, Jansen $E$ : Vitamin $D$ status and oxidative stress markers in the elderly with impaired fasting glucose and type 2 diabetes mellitus. Aging clinical and experimental research 2012, 24(6):595-602.

31. Procopio M, Borretta G: Derangement of glucose metabolism in hyperparathyroidism. Journal of endocrinological investigation 2003, 26(11):1136-1142.

32. Zeitz U, Weber K, Soegiarto DW, Wolf E, Balling R, Erben RG: Impaired insulin secretory capacity in mice lacking a functional vitamin D receptor. FASEB journal : official publication of the Federation of American Societies for Experimental Biology 2003, 17(3):509-511.

33. Carlson N, Mah R, Aburto M, Peters MJ, Dupper MV, Chen LH: Hypovitaminosis D correction and high-sensitivity Creactive protein levels in hypertensive adults. The Permanente journal 2013, 17(4):19-21.
34. Pittas AG, Harris SS, Stark PC, Dawson-Hughes B: The effects of calcium and vitamin D supplementation on blood glucose and markers of inflammation in nondiabetic adults. Diabetes care 2007, 30(4):980-986.

35. Singh U, Dasu M, Yancey P, Afify A, Devaraj S, Jialal I: Human C-reactive protein promotes oxidized low density lipoprotein uptake and matrix metalloproteinase-9 release in Wistar rats. Journal of lipid research 2008, 49(5):1015-1023.

36. Van Tits L, De Graaf J, Toenhake H, van Heerde W, Stalenhoef A: C-reactive protein and annexin A5 bind to distinct sites of negatively charged phospholipids present in oxidized low-density lipoprotein. Arteriosclerosis, thrombosis, and vascular biology 2005, 25(4):717-722.

37. Tabuchi M, Inoue K, Usui-Kataoka H, Kobayashi K, Teramoto M, Takasugi K, Shikata K, Yamamura M, Ando $\mathrm{K}$, Nishida K: The association of C-reactive protein with an oxidative metabolite of LDL and its implication in atherosclerosis. Journal of lipid research 2007, 48(4):768781.

38. Li S-F, Hu Y-W, Zhao J-Y, Ma X, Wu S-G, Lu J-B, Hu Y-R, Wang Y-C, Gao J-J, Sha Y-H: Ox-LDL upregulates CRP expression through the IGF2 pathway in THP-1 macrophages. Inflammation 2015, 38(2):576-583.

39. Taheri M, Baheiraei A, Foroushani AR, Modarres M: Resolving Vitamin D Deficiency in the Preconception Period among High-Risk Reproductive Women: A Randomized Controlled Trial. Iranian Red Crescent Medical Journal 2014, 16(1).

40. Rahnavard Z, Eybpoosh S, Homami MR, Meybodi HA, Azemati B, Heshmat R, Larijani B: Vitamin D deficiency in healthy male population: Results of the Iranian multicenter osteoporosis study. Iranian journal of public health 2010, 39(3):45. 